\title{
Optimizing the Top Quark Signal to Background Ratio at Hadron Colliders ${ }^{\dagger}$
}

\author{
R. D. Field, Y. Kanev, and M. Tayebnejad \\ Institute for Fundamental Theory, Department of Physics \\ University of Florida, Gainesville, FL 32611
}

\begin{abstract}
We investigate the event signature of the $\ell \nu b \bar{b} q \bar{q}$ decay mode of top-pair production in proton-antiproton collisions at $1.8 \mathrm{TeV}$. Neural networks and Fisher discriminates are used in conjunction with modified Fox- Wolfram "shape" variables to help distinguish the toppair signal from the $W+$ jets and $b \bar{b}+$ jets background. Instead of requiring at least four jets in the event, we find that it is faster and better to simply cut on the number of calorimeter cells with transverse energy greater than some minimum. By combining these cell cuts with the event shape information, we are able to obtain a signal to background ratio of around 9 while keeping $30 \%$ of the signal. This corresponds to a signal to background enhancement of around 370 .
\end{abstract}

$\dagger$ Supported in part by U.S. Department of Energy grant DE-FG05-86ER- -40272.

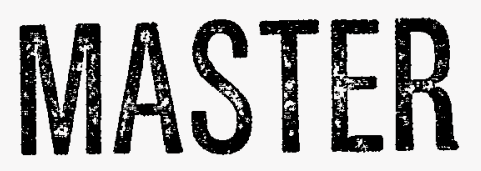




\section{DISCLAIMER}

This report was prepared as an account of work sponsored by an agency of the United States Government. Neither the United States Government nor any agency thereof, nor any of their employees, makes any warranty, express or implied, or assumes any legal liability or responsibility for the accuracy, completeness, or usefulness of any information, apparatus, product, or process disclosed, or represents that its use would not infringe privately owned rights. Reference herein to any specific commercial product, process, or service by trade name, trademark, manufacturer, or otherwise does not necessarily constitute or imply its endorsement, recommendation, or favoring by the United States Government or any agency thereof. The views and opinions of authors expressed herein do not necessarily state or reflect those of the United States Government or any agency thereof. 


\section{DISCLAIMER}

Portions of this document may be illegible in electronic image products. Images are produced from the best available original document. 


\section{Introduction}

The top quark decays into a $b$-quark and a $W$ boson $(t \rightarrow b W)$. The $W$ boson decays into a lepton ( $e$ or $\mu$ ) and a neutrino about $22 \%(2 / 9)$ of the time and into a quarkantiquark pair about $67 \%(6 / 9)$ of the time. This implies that when top-pairs are produced in hadron-hadron collisions, $p \bar{p} \rightarrow t \bar{t}+\mathrm{X}$, both of the $W$ bosons decay into a lepton and neutrino only about $5 \%$ of the time resulting in the final state consisting of two leptons, two neutrinos, and two b-quarks $(\ell \ell \nu \nu b \bar{b})$. This distinctive final state constitutes the "discovery" mode of the top quark at hadron colliders $[1,2]$. On the other hand, it is considerable more likely for one of the $W$ bosons to decay into a quark-antiquark pair resulting in a final state consisting of a lepton, a neutrino, a $b \bar{b}$, and a $q \bar{q}$ pair. The $\ell \nu b \bar{b} q \bar{q}$ mode shown in Fig. 1a occurs about $35 \%$ of the time or about 7 times more often than the purely leptonic mode. The backgrounds are larger for this decay mode, but so is the signal. When each of the four outgoing quarks produce a distinct jet, then the resulting event contains a lepton, a neutrino, and four jets $(\ell \nu j j j j)$. This decay mode is used to analyze the properties of the top quark in more detail and to determine, for example, the top mass [3-5]. The purely hadron six jet decay mode occurs about $60 \%$ of the time, but it is buried underneath "ordinary" QCD multijet production.

In this paper, we concentrate on the $\ell \nu b \bar{b} q \bar{q}$ decay mode of top-pair production in proton-antiproton collisions at $1.8 \mathrm{TeV}$ and investigate ways to optimize this signal over the backgrounds. The event topology of the signal is shown in Fig. 1b and consists of a lepton, a neutrino, and four outgoing quarks which manifest themselves as "jets". In the center-of-mass of a $175 \mathrm{GeV}$ top quark, the $W$ boson and $b$-quark decay products each have a momentum of about $70 \mathrm{GeV}$. Furthermore, in the CM frame of the $W$ boson, the quark and antiquark decay products each have a momentum of about $40 \mathrm{GeV}$. The top-pair are produced near threshold resulting in a typical event that is rather spherical in shape with all six decay products, $\ell \nu b \bar{b} q \bar{q}$, having large transverse energy. The background comes from the "ordinary" QCD production of large transverse momentum $W$ bosons plus multiple jets as shown in Fig 2a and from the production of $b$-quark pairs plus associated jets as illustrated in Fig. 2b. We begin our analysis of the signal and backgrounds in Section II 
(a) Top-Pair Production

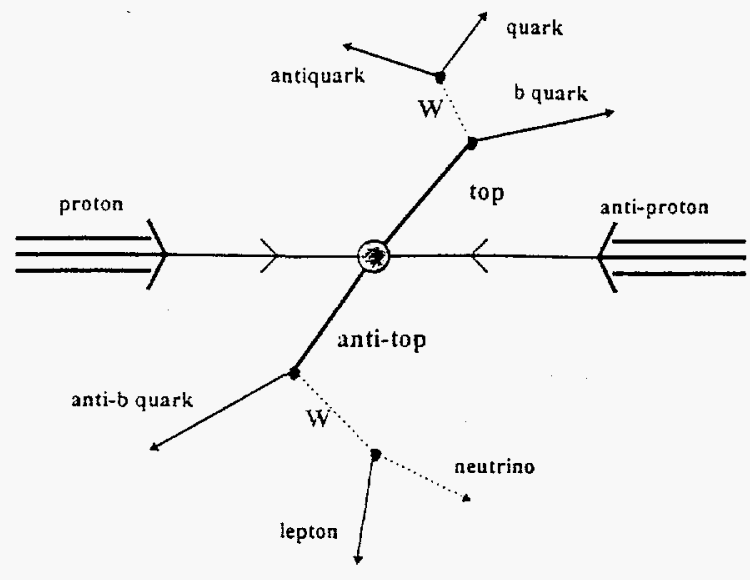

(b) Top-Pair Event Topology

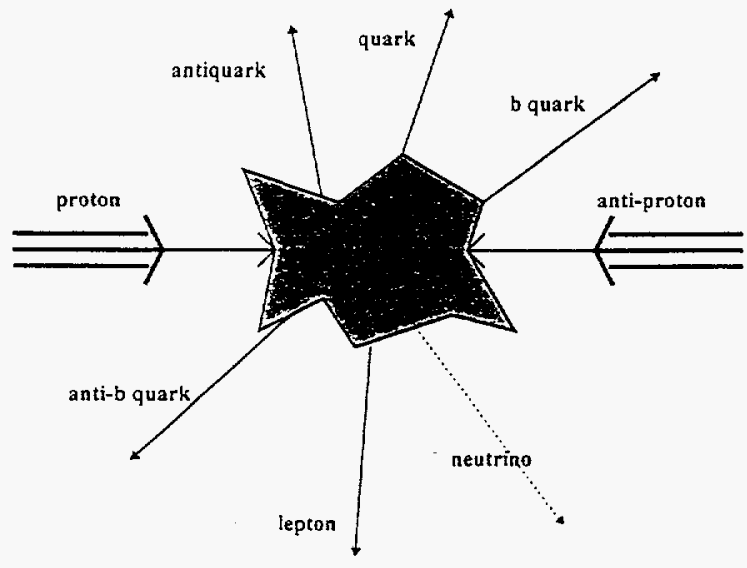

Figure 1 (a) Illustration of top-pair production in proton- antiproton collisions in which one of the $W$ bosons decays leptonically and the other decays hadronically resulting in a final state consisting of a lepton, a neutrino, plus a $b \vec{b}$ and a $q \bar{q}$ pair. (b) Shows the event topology for the top-pair signal. If each outgoing quark produces a distinct jet then the final state containes a lepton, a neutrino (missing $E_{T}$ ), and four jets.

with a discussion of the data selection and initial cuts. In Section III, we reconstruct the invariant mass of the top-pair and compare it with the true parton-parton CM energy. In Section IV, we introduce modified Fox-Wolfram "shape" variables and apply them to 
(a) $w+$ jets background

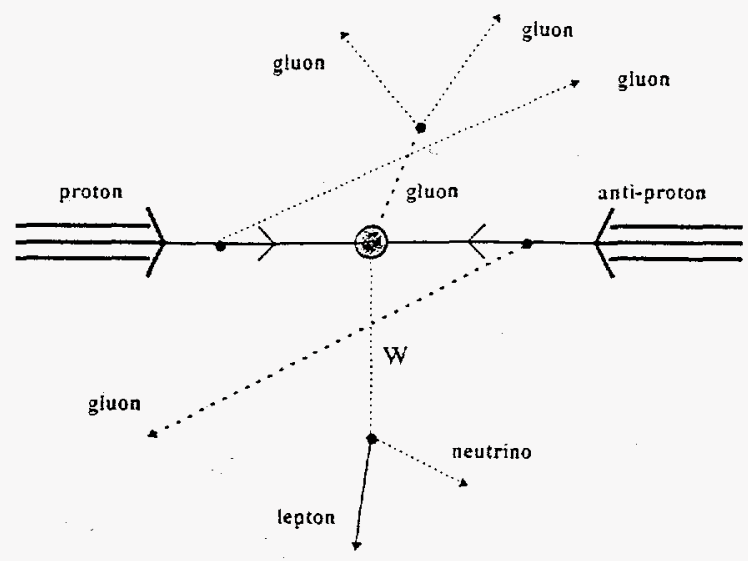

(b) b-pair + jets background

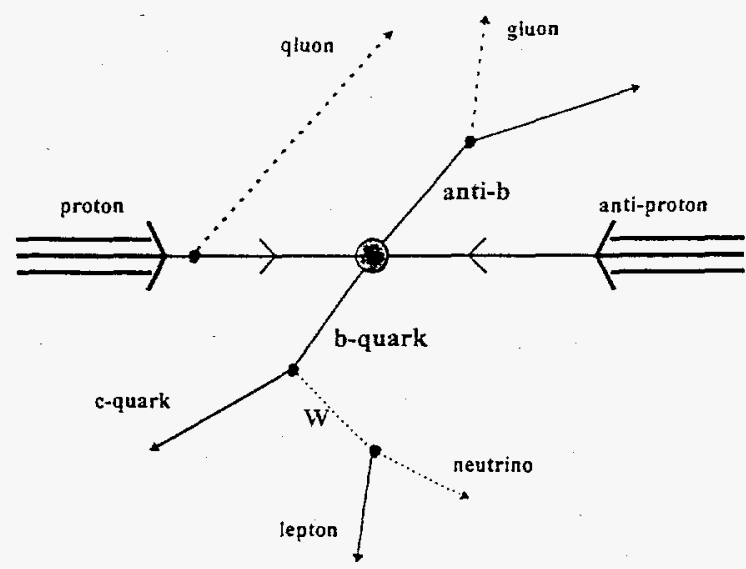

Figure 2 Illustration the (a) $W+$ jets background and the (b) $b \bar{b}+$ jets background to the top-pair production in proton-antiproton collisions shown in Fig. 1.

the outgoing jets and in Section $V$ the use of neural networks and Fisher discriminates is explored. Section VI is reserved for summary and conclusions. 


\section{Event Generation, Data Selection and Cuts}

ISAJET version 7.06 is used to generate top quarks with a mass of $175 \mathrm{GeV}$ in $1.8 \mathrm{TeV}$ proton-antiproton collisions. At this energy, $175 \mathrm{GeV}$ top-pairs are produced via quarkantiquark annihilation, $q \bar{q} \rightarrow t \bar{t}$, about $88 \%$ of the time and by gluon-gluon fusion, $g g \rightarrow t \bar{t}$, the remaining $12 \%$. We refer to this as the "signal". We have normalized the top cross section to be $7.5 \mathrm{pb}$ corresponding to 750 events with an integrated luminosity of $100 / \mathrm{pb}$ [35]. The "background" consists of single $W$ boson events generated with the hard-scattering transverse momentum, $\hat{k}_{T}$, greater than $25 \mathrm{GeV}$. Single $W$ bosons are produced at large transverse momentum via the "ordinary" $\mathrm{QCD}$ subprocesses $q g \rightarrow W q, \bar{q} g \rightarrow W \bar{q}$, and $q \bar{q} \rightarrow W g$. These subprocesses, of course, generate addition gluons via bremsstrahlung off both incident and outgoing color non-singlet partons, resulting in multiparton final states which subsequently fragment into hadrons. This is referred to as the $W+$ jets background. Another background is b-quark pairs produced via the subprocesses $q \bar{q} \rightarrow b \bar{b}$ and $q \bar{q} \rightarrow b \bar{b}$ plus the accompanying radiation. This is referred to as the $b \bar{b}+$ jets background.

We do not attempt to do a detailed simulation of the CDF or D0 detector [3,4]. Events are analyzed by dividing the solid angle into "calorimeter" cells having size $\Delta \eta \Delta \phi=$ $0.1 \times 7.5^{\circ}$, where $\eta$ and $\phi$ are the pseudorapidity and azimuthal angle, respectively. Our simple calorimeter covers the range $|\eta|<4$ and has 3840 cells. A single cell has an energy (the sum of the energies of all the particles that hit the cell excluding neutrinos) and a direction given by the coordinates of the center of the cell. From this the transverse energy of each cell is computed from the cell energy and direction. We have taken the energy resolution to be perfect, which means that the only resolution effects are caused by the lack of spatial resolution due to the cell size. Large transverse momentum leptons are analyzed separately and are not included when computing the energy of a cell.

\section{Lepton plus Missing Transverse Energy Trigger}

The "zero-level" trigger is designed to select large transverse momentum $W$ bosons that have decayed into a charged lepton and a neutrino. This first cut is made by demanding that the event contain at least one isolated high transverse momentum charged 
lepton $\left(\ell^{ \pm}=e^{ \pm}\right.$or $\left.\mu^{ \pm}\right)$in the central region satisfying:

- $P_{T}\left(\ell^{ \pm}\right)>15 \mathrm{GeV},\left|\eta\left(\ell^{ \pm}\right)\right|<2.5$.

"Isolated" leptons are defined by demanding that the total transverse energy within a distance $R_{\ell}$ of the lepton in $\eta-\phi$ space be less than $E_{T}^{\ell}(\max )$. For this analysis,

- $R_{\ell}=0.2, E_{T}^{\ell}(\max )=5 \mathrm{GeV}$.

In addition, the event must have large missing transverse energy, $\mathbb{E}_{T}$, and an overall lepton-neutrino transverse momentum, $P_{T}(\ell \nu)$, given by:

- $E_{T}>20 \mathrm{GeV}, P_{T}(\ell \nu)>25 \mathrm{GeV}$

where the missing transverse momentum 2 -vector, $\vec{p}_{T}$, is determined from the transverse energy grid (i.e., the calorimeter) and

$$
\begin{aligned}
\mathscr{E}_{T}^{2} & =\not p_{x}^{2}+\not p_{y}^{2}, \\
P_{T}^{2}(\ell \nu) & =\left(p_{x}^{\ell}+\not p_{x}\right)^{2}+\left(p_{y}^{\ell}+\not p_{y}\right)^{2}
\end{aligned}
$$

where the $\hat{x}$-axis and $\hat{y}$-axis are perpendicular to the colliding beams and the $\hat{z}$-axis is parallel.

This selection of $P_{T}\left(\ell^{ \pm}\right)>15 \mathrm{GeV}, \mathbb{E}_{T}>20 \mathrm{GeV}$, and $P_{T}(\ell \nu)>25 \mathrm{GeV}$ is referred to as the lepton plus missing $E_{T}$ trigger. Table 1 shows that about 165 top-pair events survive this selection criterion (22\% of the overall top signal). For illustration, we take the integrated luminosity to be $100 / \mathrm{pb}$. Table 1 also shows that about $7,000 W+$ jets and roughly $500 b \bar{b}+$ jets background events also survive this "zero-level" cut. The lepton isolation requirements do a good job removing most of the $b \bar{b}+$ jets background, so we will concentrate primarily on the $W+$ jets background. At this stage, the background and is about 43 times the signal.

In order to quantify how various additional cuts enhance the signal above the background, we define the enhancement, $F_{e n h}$, and the efficiency, $F_{\text {eff }}$, of a given set of cuts as follows:

$$
\begin{aligned}
& F_{\text {enh }}=\frac{\% \text { of signal surviving cut }}{\% \text { of background surviving cut }} \\
& F_{\text {eff }}=\% \text { of signal surviving cut. }
\end{aligned}
$$




\begin{tabular}{|c|c|c|c|c|c|c|c|c|c|}
\hline Cut or Selection & $\begin{array}{l}\text { Top } 117 \\
\text { Events }\end{array}$ & $\begin{array}{l}75 \mathrm{GeV}) \\
\mid \begin{array}{r}\text { Effici } \\
\text { Relative }\end{array}\end{array}$ & $\begin{array}{l}\text { Signal } \\
\text { iency } \\
\text { Overail }\end{array}$ & $\begin{array}{c}\text { W+jet } \\
\text { Events }\end{array}$ & \begin{tabular}{|} 
is Backg \\
Effici \\
Relative
\end{tabular} & $\begin{array}{l}\text { round } \\
\text { ency } \\
\text { Overall }\end{array}$ & $\begin{array}{l}\text { Enhanc } \\
\text { Relative }\end{array}$ & $\begin{array}{l}\text { ement } \\
\text { Overall }\end{array}$ & Sig/Bak \\
\hline $\begin{array}{l}\text { "Zero-Level" Lepton } \\
\text { plus Missing ET } \\
\text { Trigger: } \\
P_{\text {T(l) }}>15 \mathrm{GeV} \\
E_{\mathrm{T}}(\text { miss })>20 \mathrm{GeV} \\
P_{\mathrm{r}}(\text { (Iv })>25 \mathrm{GeV}\end{array}$ & 165 & $100 \%$ & $100 \%$ & $\begin{array}{l}7,044 \\
(470)\end{array}$ & $100 \%$ & $100 \%$ & 1.0 & 1.0 & 0.0234 \\
\hline $\begin{array}{l}\text { Calorimeter Cell } \\
\text { Cuts: } \\
N \text { (cell) }>7\left(E_{\bar{T}}>5\right) \\
E_{T}(\text { sum })>100 \mathrm{GeV}\end{array}$ & 113 & $69 \%$ & $69 \%$ & $\begin{array}{l}49 \\
(3)\end{array}$ & $0.7 \%$ & $0.7 \%$ & 99.7 & 99.7 & 2.3 \\
\hline $\begin{array}{l}\text { Fisher Cut: } \\
F>0.75\end{array}$ & 49 & $44 \%$ & $30 \%$ & 6 & $12 \%$ & $0.1 \%$ & 3.7 & 373.0 & 8.7 \\
\hline
\end{tabular}

Table $1175 \mathrm{GeV}$ Top quark pairs produced in $1.8 \mathrm{TeV}$ proton-antiproton collisions. The table shows the number of events (with $\mathcal{L}=100 / \mathrm{pb}$ ) for the top-pair signal and the $W+$ jets background. The $b \dot{b}+$ jets backgrond is shown in paranthesis. The "zero-level" lepton plus missing $E_{T}$ trigger is used as a reference point and is normalized to $100 \%$. The enhancement factor is defined to be the percentage of signal divided by the percentage of background surviving the given set of cuts. Both the overall and relative enhancement factors are shown.

We define the "zero-level" trigger to be the reference point and the fraction of events escaping this cut is set to $100 \%$ in Table 1 . Similarly, all "enhancement" factors are set to one at this level as we measure the effectiveness of all other additional cuts from this point. The overall enhancement and efficiency is determined relative to the "zero-level" trigger, while relative enhancements and efficiencies are determined by examining the number of events before and after the particular cut.

\section{Calorimeter Cell Cuts}

At this stage in the analysis, one normally demands that the event contain at least four jets [3-5]. Cutting on the number of jets is a way to preferencially select the top-pair signal over the background. However, we have found that it is faster and better to simply cut on the number of calorimeter cells, $N_{\text {cell }}$, with transverse energy greater than some minimum, $E_{T}^{c e l l}(\mathrm{~min})$. Fig. 3 shows the calorimeter cell multiplicity with $E_{T}^{\text {cell }}(\mathrm{min})=5 \mathrm{GeV}$ for the top-pair signal and the $W+$ jets background. On the average, the top-pair signal populates 


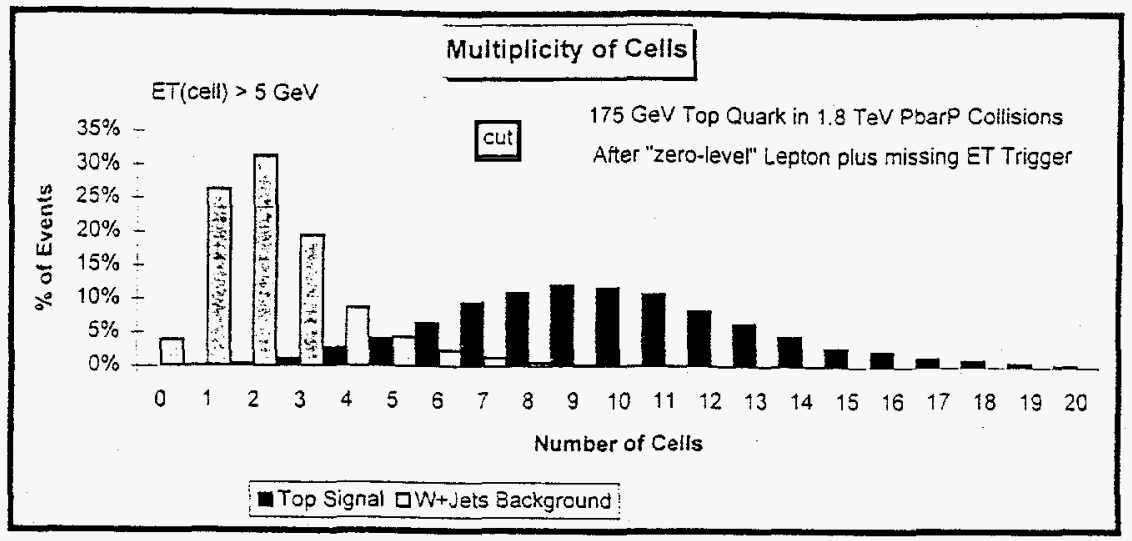

Figure 3 Shows the multiplicity of calorimeter cells containing at least $5 \mathrm{GeV}$ of transverse energy for the of the top-pair signal and the $W$ +jets background. In all cases, the events have survived the "zero-level" lepton plus missing $E_{T}$ trigger. The plot shown the percentage of events with $N$ cells with $E_{T}($ cell $)>5 \mathrm{GeV}$. The position of our cell cut is marked by the dotted line.

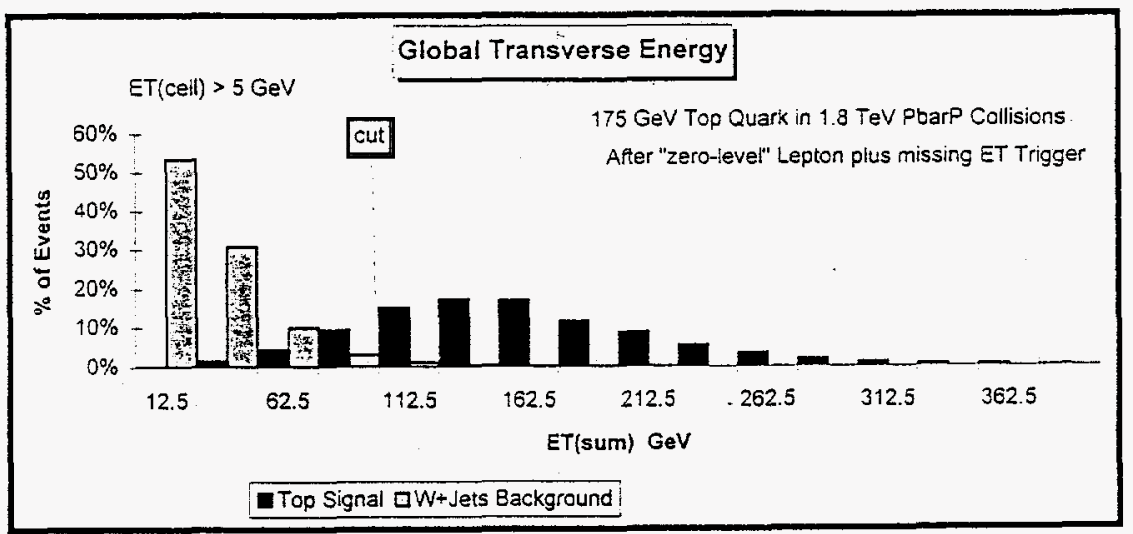

Figure 4 Shows the total transverse energy of all the calorimeter cells with $E_{T}($ cell $)>5 \mathrm{GeV}$ for the of the top-pair signal and the $W+$ jets background. In all cases, the events have survived the "zero-level" lepton plus missing $E_{T}$ trigger. The plot shown the percentage of events with $E_{T}(s u m)$ within a $25 \mathrm{GeV}$ bin. The position of our cell cut is marked by the dotted line. 
a larger number of cells than does the background. Obviously this is because the top-pair signal produces more jets, however, one does not have to define a "jet" to see this topology. The top-pair signal produces transverse energy flying out in all directions and this can be seen directly from the calorimeter cell multiplicity.

The top-pair signal also produces more global transverse energy than the background. This is shown in Fig. 4, where we define $E_{T}$ (sum) to be the sum of the transverse energy of all the calorimeter cells with $E_{T}>E_{T}^{\text {cell }}(\min )$. As illustrated in Fig. 3 and Fig. 4, we make the following calorimeter cell cuts:

- $N_{\text {cell }} \geq 8$ with $E_{T}^{\text {cell }}(\mathrm{min})=5 \mathrm{GeV}$, and $E_{T}(\mathrm{sum})>100 \mathrm{GeV}$.

At this stage one could cut harder on $E_{T}$ (sum) and remove more background. However, we want to avoid as much as possible cuts that cause the background invariant mass to peak at the same place as the top-pair signal. For this reason, we will use event "shape" variables to further improve the signal to background ratio.

Table 1 shows that of the 165 top-pair events passing the "zero-level" lepton plus missing $E_{T}$ cut roughly $69 \%$ also pass the calorimeter cell cuts. On the other hand, less than $1 \%$ of the $W+$ jets background events survive the cell cuts. The calorimeter cell cuts produce an enhancement of $0.69 / 0.007$ or about 100 over the $W+$ jets background with a $69 \%$ efficiency, resulting in a signal to background ratio of about 2 . The $N_{\text {cell }} \geq 8$ with $E_{T}^{\text {cell }}(\min )=5 \mathrm{GeV}$ cut produces more than a factor of two better enhancement than the traditional "jet cuts" (i.e., $N_{\text {jet }} \geq 4$ ). Adding the $E_{T}(\mathrm{sum})>100 \mathrm{GeV}$ cut gives an additional relative enhancement of more than a factor of three.

\section{Reconstructing the Top-Pair Invariant Mass}

\section{Reconstructing the neutrino momentum}

Ideally one would like to reconstruct the invariant mass of the top-pair from its decay products: lepton, neutrino, and four jets. However, the neutrino is not detected and its presence must be inferred by examining the missing transverse momentum, $\vec{p}_{T}$. If we set the transverse momentum components of the neutrino equal to the missing transverse 


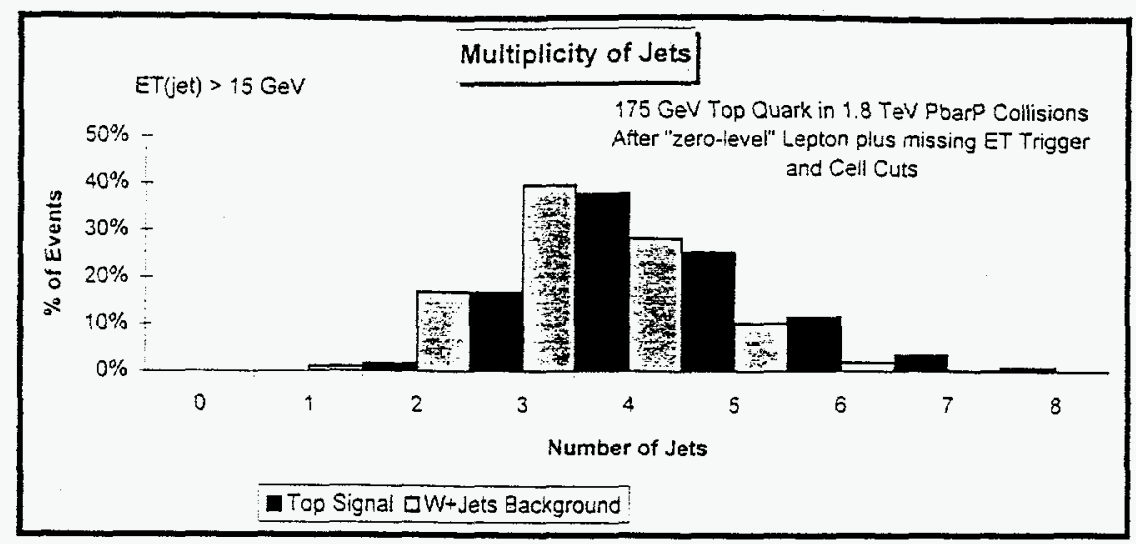

Figure 5 Shows the multiplicity of jets with transverse energy greater than $15 \mathrm{GeV}$ for the of the top-pair signal and the $W+$ jets background. In all cases the events have survived the "zero-level" lepton plus missing $E_{T}$ trigger and the calorimeter cells cuts. The plot shown the percentage of events with $N$ jets with $E_{T}$ (jet) $>15 \mathrm{GeV}$.

momentum,

$$
\vec{p}_{T}^{\nu}=\vec{p}_{T}
$$

and assume that the charged lepton and the neutrino are the result of a $W$ decay (and neglect the $W$ width) then the longitudinal momentum of the neutrino is given by one of the two solutions:

$$
p_{L}^{\nu}=\left[A p_{L}^{\ell} \pm E_{\ell}^{2} \sqrt{A^{2}-4\left(p_{T}^{\ell}\right)^{2}\left(p_{T}^{\nu}\right)^{2}}\right] / 2\left(p_{T}^{\ell}\right)^{2}
$$

where $E_{\ell}, p_{L}^{\ell}$, and $p_{T}^{\ell}$, are the energy, longitudinal momentum, and transverse momentum, respectively, of the charged lepton, and $p_{T}^{\nu}$ is the transverse mementum of the neutrino. The quantity $A$ is given by

$$
A=M_{W}^{2}+2 \vec{p}_{T}^{\ell} \cdot \vec{p}_{T}^{\nu}=M_{W}^{2}+p_{T}^{\ell} p_{T}^{\nu} \cos \phi
$$

where $\phi$ is the azimuthal angle between the transverse momentum vector of the charged lepton and the neutrino. We include both solutions in our determination of the top-pair invariant mass. 
Adding in the momentum and energy of the jets

We have not used jets in our event trigger, however, we do use jets to reconstruct the top-pair invariant mass. In addition, we use the jet topology to help further distinguish the signal from the backgrounds. Jets are defined using a simple algorithm. One first considers the "hot" cells (those with transverse energy greater than $5 \mathrm{GeV}$ ). Cells are combined to form a jet if they lie within a specified "radius" $R_{j}^{2}=\Delta \eta^{2}+\Delta \phi^{2}$ in $\eta-\phi$ space from each other. Jets have an energy given by the sum of the energy of each cell in the cluster and a momentum $\vec{p}_{j}$ given by the vector sum of the momentums of each cell. The invariant mass of a jet is simply $M_{j}^{2}=E_{j}^{2}-\vec{p}_{j} \cdot \vec{p}_{j}$. In this analysis, we take the jet radius to be $R_{j}=0.4$ and require jets to have at least $15 \mathrm{GeV}$ of transverse energy. Namely,

$$
\text { - } R_{j}=0.4 \text { and } E_{T}(j e t)>15 \mathrm{GeV} \text {. }
$$

The top-pair invariant mass, $M_{t \bar{t}}$, is constructed from the energy and momentum of the charged lepton, the energy and momentum of the reconstructed neutrino, and the overall momentum vector of the associated jets as follows:

$$
M_{t \bar{t}}^{2}=\left(E_{\ell}+E_{\nu}+E_{j e t s}\right)^{2}-\left(\vec{p}_{\ell}+\vec{p}_{\nu}+\vec{p}_{j e t s}\right)^{2},
$$

where

$$
\vec{p}_{j e t s}=\sum_{i}^{j e t s} \vec{p}_{i}
$$

and

$$
E_{j e t s}=\sum_{i}^{j e t s} E_{i} .
$$

The overall jet energy, $E_{j e t s}$, and momentum, $\vec{p}_{j e t s}$, is constructed by summing over all jets with transverse energy greater than $15 \mathrm{GeV}$. We do not require the event to have a minimum of four jets. The calorimeter cell cuts have replaced the need to make a jet multiplicity cut. This can be seen in Fig. 5 which shows the multiplicity of jets with $E_{T}(j e t)>15 \mathrm{GeV}$ for the top-pair signal and the $W+$ jets background after the lepton plus missing $E_{T}$ trigger and the calorimeter cell cuts. The cell cuts have forced the signal and background jet multiplicities to look similar and one does not gain much by making 


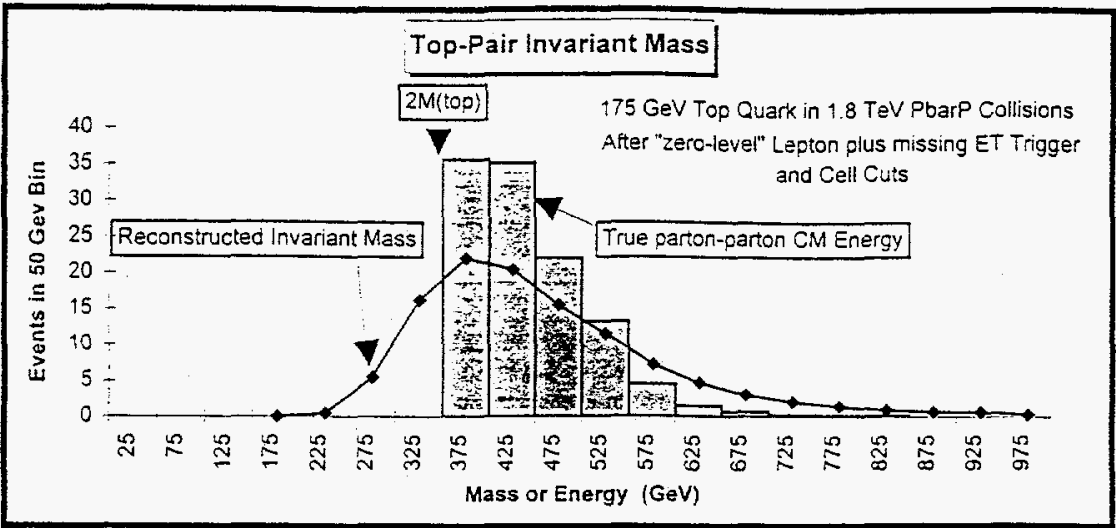

Figure 6 Shows the reconstructed top-pair invariant mass, $M_{t \bar{t}}$, for $175 \mathrm{GeV}$ top quarks produced in $1.8 \mathrm{TeV}$ proton-antiproton collisions (solid curve). The plot contains only the top-pair signal and corresponds to the number of events per year (with $\mathcal{L}=100 / \mathrm{pb}$ ) in a $50 \mathrm{GeV}$. The events have survived the "zero-level" lepton plus missing $E_{T}$ trigger and the calorimeter cell cuts. Also shown in the true parton-parton CM energy of the event (not directly observable experimentally).

an additional jet multiplicity cut. (At this stage, requiring $N_{\text {jet }} \geq 4$ would result in an additional relative enhancement of about 2 with an efficiency of $81 \%$.)

\section{Comparing with the parton-parton CM energy}

The top-pair invariant mass, $M_{t \bar{t}}$, corresponds to the center-of-mass energy, $\hat{E}_{c m}$, of the underlying parton-parton two-to-two subprocess which has a threshold at twice the mass of the top quark, $\hat{E}_{c m} \geq 2 M_{\text {top }}$. This is seen clearly in Fig. 6 which compares the true $q \bar{q} \rightarrow t \bar{t}$ and $g g \rightarrow t \bar{t} \mathrm{CM}$ energy, $\hat{E}_{c m}$ (not experimentally observable), with the reconstructed top-pair invariant mass, $M_{t \bar{t}}$. If the neutrino momentum could be precisely determined from the missing $E_{T}$ and if we knew exactly which particles to include in the jets then the two curves in Fig. 6 would agree. Although one cannot precisely reconstruct the parton-parton CM energy, there still remains a nice peak in the reconstructed top-pair invariant mass at twice the top mass. In this paper, we use the observation of this peak as a measure of how well one can determine the top quark mass and we would like to remove as much background as possible from the peak. 


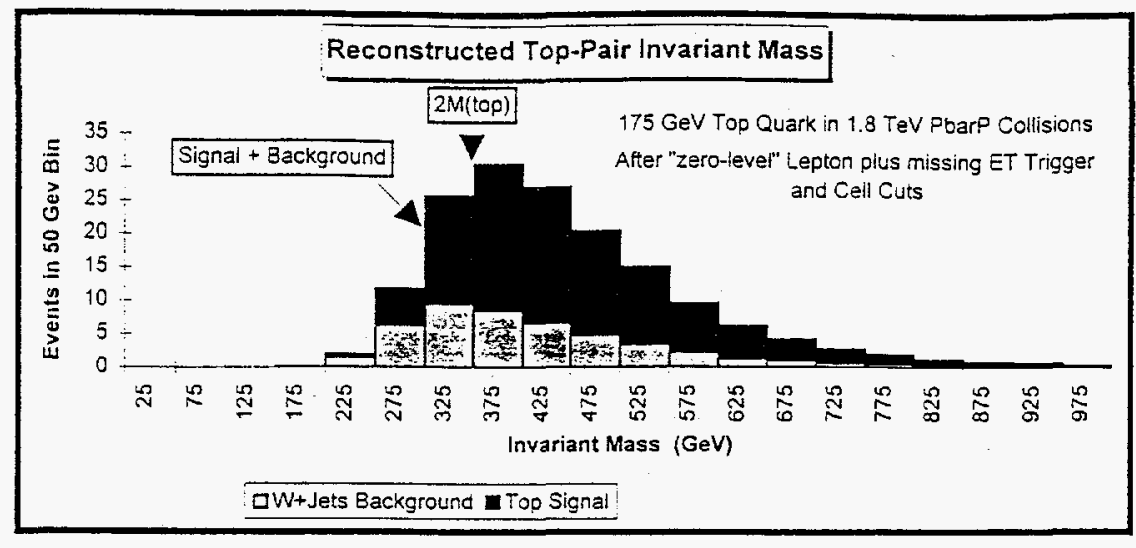

Figure 7 Shows the reconstructed top-pair invariant mass, $M_{t \bar{t}}$, for $175 \mathrm{GeV}$ top quarks produced in $1.8 \mathrm{TeV}$ protonantiproton collisions together with the $W+$ jets background. The plot shown the sum of the signal plus background and corresponds to the number of events per year (with $\mathcal{L}=100 / \mathrm{pb}$ ) in a $50 \mathrm{GeV}$. The events have survived the "zero-level" lepton plus missing $E_{T}$ trigger and the calorimeter cell cuts.

Fig. 6 includes only the top-pair signal with no background. Fig. 7 shows the reconstructed parton-parton CM energy for the top-pair signal and the $W+$ jets background after the "zero level" lepton plus missing $E_{T}$ trigger and the calorimeter cell cuts. The plot shown the sum of the signal and the background. At this stage the signal is about twice the background. However, the signal to background ratio can be improved by examining in more detail the "shape" of the events.

\section{Variables that Characterize the Event Shape}

\section{Fox-Wolfram Moments}

In 1979 Geoffrey Fox and Stephen Wolfram [6] constructed a complete set of rotationally invariant observables, $H_{\ell}$ which can be used to characterize the "shapes" of the final states in electron-positron annihilations. They are constructed from the momentum 


\begin{tabular}{|c|c|c|c|c|c|}
\hline & \multicolumn{4}{|c|}{ Top(175 GeV) Signal Z Z Jets Background } & \\
\hline Moment & mean & stdev & mean & stdey & Fisher Coefficient \\
\hline $\mathrm{H}_{1}$ & 0.24 & 0.18 & 0.36 & 0.25 & -0.500 \\
\hline $\mathrm{H}_{2}$ & 0.28 & 0.16 & 0.44 & 0.22 & -1.282 \\
\hline $\mathrm{H}_{3}$ & 0.28 & 0.14 & 0.40 & 0.19 & -1.088 \\
\hline $\mathrm{H}_{4}$ & 0.28 & 0.13 & 0.41 & 0.18 & -0.978 \\
\hline $\mathrm{H}_{5}$ & 0.29 & 0.12 & 0.40 & 0.17 & -0.544 \\
\hline$\overline{\mathrm{H}_{6}}$ & 0.29 & 0.12 & 0.40 & 0.17 & -0.069 \\
\hline
\end{tabular}

Table 2 Shows the mean value and standard deviation from the mean of six of the Modified Fox-Wolfram moments applied to the jets in the event with transverse energy greater than $15 \mathrm{GeV}$. Results are shown for the top-pair signal and the $W+$ jets background. Also shown are the resulting Fisher coefficients.

vectors, $\vec{p}$, of all the final state particles as follows,

$$
H_{\ell}=\left.\left.\left(\frac{4 \pi}{2 \ell+1}\right) \sum_{m=-\ell}^{+\ell}\right|_{i} ^{\text {particles }} \sum_{\ell}^{m}\left(\Omega_{i}\right) \frac{\left|\vec{p}_{i}\right|}{E_{t o t}}\right|^{2}
$$

where the inner sum is over the particles produced and $Y_{\ell}^{m}$ are the spherical harmonics. Here one must choose a particular set of axes to evaluate the angles, $\Omega_{i}=\left(\theta_{i}, \phi_{i}\right)$, of the final state particles, but the values of the $H_{\ell}$ are independent of this choice. These moments lie in the range $0 \leq H_{\ell} \leq 1$ and if energy conserved in the final state then $H_{0}=1$ (neglecting the masses). If momentum is conserved in the final state then $H_{1}=0$.

The Fox-Wolfram observables (or moments) constitute a complete set of shape parameters. For example, the collinear "two-jet" final state results in $H_{\ell} \approx 1$ for even $\ell$ and $H_{\ell} \approx 0$ for odd $\ell$. Events that are completely spherically symmetric give $H_{\ell} \approx 0$ for all $\ell$.

\section{Modified Fox-Wolfram Moments Applied the Jets}

In hadron-hadron collisions spherical symmetry is lost and we are interested more in the shape of events in the transverse plane. For example, the Fox-Wolfram moments when applied directly to hadron-hadron collisions would interpret a minimum bias event as a 


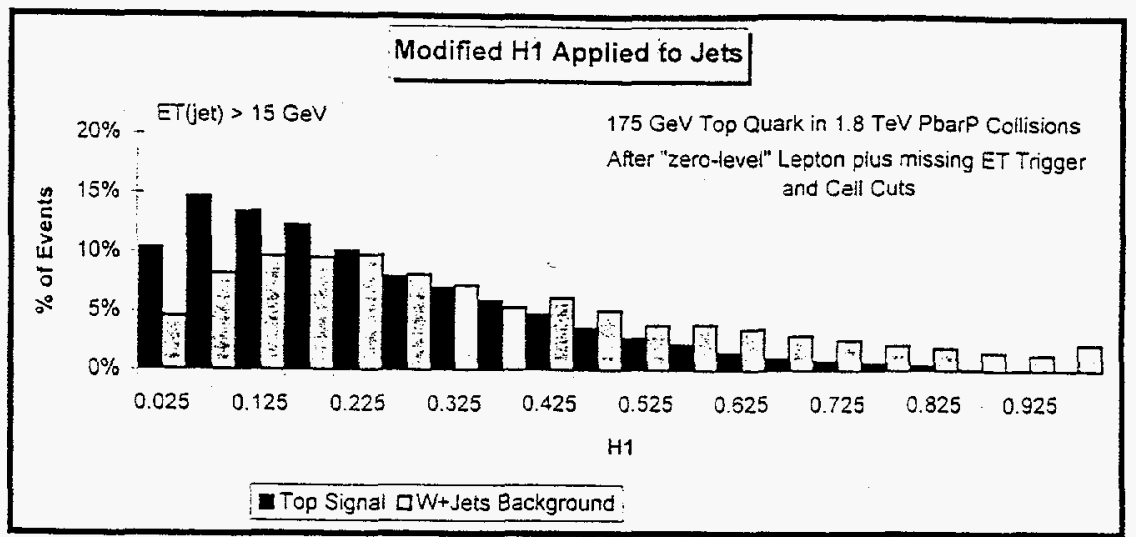

Figure 8 Shows the modified Fox-Wolfram moment, $\hat{H}_{1}$, calculated using the jets in the event with transverse energy greater than $15 \mathrm{GeV}$ for top-pair signal and for the $W$ +jets background. The plot showns the percentage of events in a $0.05 \mathrm{bin}$. The events have survived the "zero- level" lepton plus missing $E_{T}$ trigger and the calorimeter cell cuts. (If the vector sum of the monentum of all the jets in the events is zero then $\hat{H}_{1}=0$.)

"two-jet" event. Whereas, we would like to have a minimum bias event treated more like a spherically symmetric $e^{+} e^{-}$final state (i.e., no structure). To accomplish this, we define the following modified Fox-Wolfram moments for hadron-hadron collisions,

$$
\hat{H}_{\ell}=\left(\frac{4 \pi}{2 \ell+1}\right) \sum_{m=-\ell}^{+\ell}\left|\sum_{i}^{j e t s} Y_{\ell}^{m}\left(\Omega_{i}\right) \frac{E_{T}^{i}}{E_{T}(\mathrm{sum})}\right|^{2},
$$

where the inner sum is now over all the jets in the event with transverse energy, $E_{T}^{i}$, greater than $15 \mathrm{GeV}$ and $\Omega_{i}=\left(\theta_{i}, \phi_{i}\right)$ the angular location jet. Here, $E_{T}(s u m)$ is the sum of the transverse energy of all the jets that are included in the sum. These modified moments also lie in the range $0 \leq \hat{H}_{\ell} \leq 1$ and by definition $\hat{H}_{0}=1$. Furthermore, if the transverse momentum of the jets in the event is conserved then $\hat{H}_{1}=0$. In this case, however, events that are "cylindrically symmetric" about the beam axis give $\hat{H}_{\ell} \approx 0$ for all $\ell$.

Table 2 shows the mean values and standard deviations for six of the modified FoxWolfram moments calculated using all jets with $E_{T}(j e t)>15 \mathrm{GeV}$ for events that have 


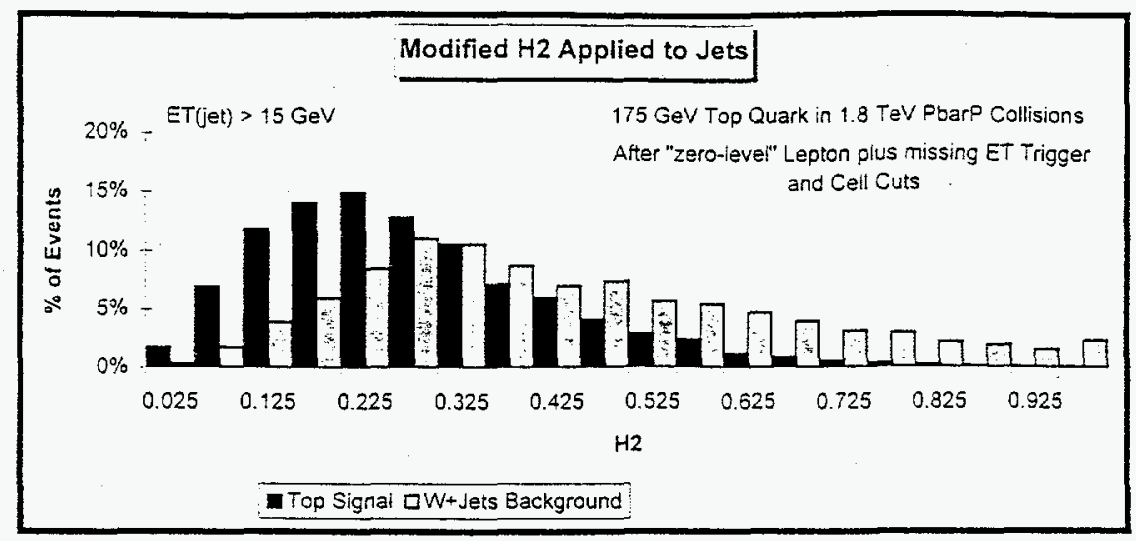

Figure 9 Shows the modified Fox-Wolfram moment, $\hat{H}_{2}$, calculated using all the jets in the event with transverse energy greater than $15 \mathrm{GeV}$ for top-pair signal and for the $W+$ jets background. The plot showns the percentage of events in a $0.05 \mathrm{bin}$. The events have survived the "zero- level" lepton plus missing $E_{T}$ trigger and the calorimeter cell cuts.

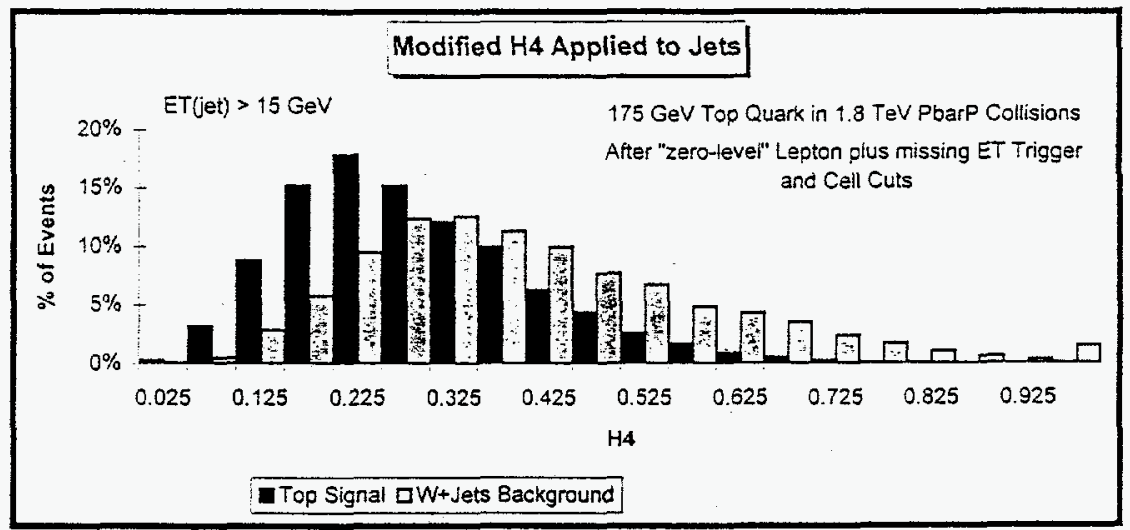

Figure 10 Shows the modified Fox-Wolfram moment, $\hat{H}_{4}$, calculated using all the jets in the event with transverse energy greater than $15 \mathrm{GeV}$ for top-pair signal and for the $W$ +jets background. The plot showns the percentage of events in a 0.05 bin. The events have survived the "zero- level" lepton plus missing $E_{T}$ trigger and the calorimeter cell cuts. 
survived the "zero-level" lepton and missing $E_{T}$ trigger and the calorimeter cell cuts. There are clearly still some differences between the jet topologies of the top-pair signal and the $W+$ jets background. The mean values of the six moments $\hat{H}_{1}, \ldots, \hat{H}_{6}$ are smaller for the signal than the background indicating that the jets originating from the top- pair signal form a more cylindrically symmetric pattern when they emerge from the event than do the background jets. The top-pair jets are more spread out in $\eta$ - $\phi$ space. This can be seen in Figs. 8, 9 , and 10 which show the $\hat{H}_{1}, \hat{H}_{2}$, and $\hat{H}_{4}$ distributions, respectively, for the signal and background. At this stage one could simply make a linear cut on, for example, $\hat{H}_{2}$. Requiring $\hat{H}_{2}<0.3$ gives an additional signal to background enhancement of about 2 with a relative efficiency of around $60 \%$. One can do a little better, however, by using the information of all six of the $\hat{H}_{\ell}$ 's simultaneously. This can be done by constructing a neural network or by using Fisher discriminates.

\section{Neural Network Analysis}

Neural networks are an excellent tool for separating patterns into categories (e.g., signal and background). Our neural networks [7] consist of a set of $N_{\text {in }}$ inputs, $\{x\}$, which can have any value and one output, $z_{n e t}$, which is restricted to the range, $0<z_{n e t}<1$. The net output is a function of the input set $\{x\}$ and the network "memory" parameters as follows:

$$
z_{\text {net }}=F_{\text {net }}(\{x\},\{w\},\{T\})
$$

where the network memory consists of a set of weights, $\{w\}$, and a set of thresholds $\{T\}$. The goal is to construct a network that can distinguish between two patterns of input data, "signal" events and "background" events, where each event is characterized by the $N_{\text {in }}$ variables. A "perfect" network responds with $z_{n e t}$ near one for a signal input and with $z_{\text {net }}$ near zero for a background input and a single cut can be made on this network output which will enhance the signal over the background.

\section{Network Inputs and Training}

Of course, the key to a good network lies in the selection of the input variables. These 


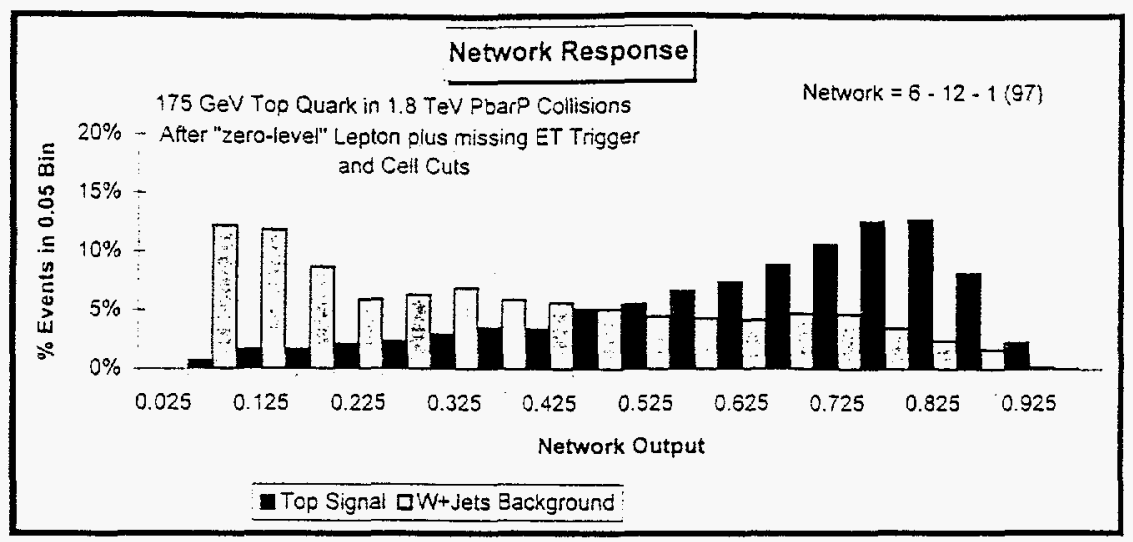

Figure 11 Shows the network response, $z_{\text {net }}$, for the sample of signal and background events used in the training. The plot corresponds to the percentage of events with $z_{n e t}$ within a 0.05 bin for the top-pair signal and the $W+$ jets background. The events have survived the "zero-level" lepton plus missing $E_{T}$ trigger and the calorimeter cell cuts.

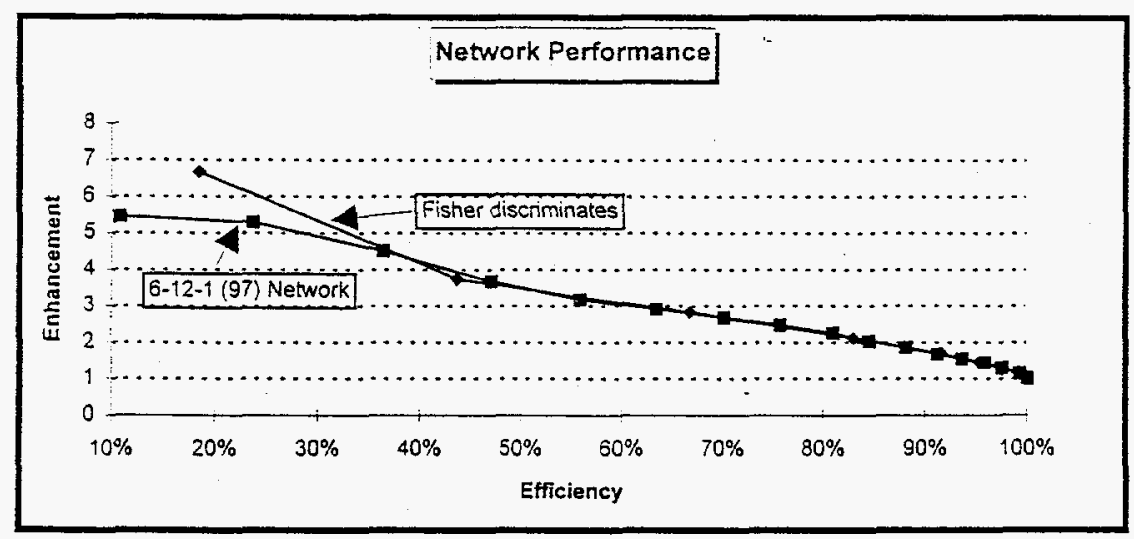

Figure 12 Shows the enhancement versus the efficiency for the training sample of events for a 6-12-1 neural network with 97 memory parameters. Each point in the plot corresponds to a different choice for the network cut-off with the lower efficiencies and higher enhancements corresponding to larger values of $z_{\text {cut }}$. The network enhancements are compared with the enhancements arrived at by the use of Fisher discriminates.

variables must characterize the differences between the signal and the background. We 
choose the first six modified Fox-Wolfram variables (applied to the jets) as the network inputs:

$$
\begin{array}{ll}
x_{1}=\hat{H}_{1}, & x_{2}=\hat{H}_{2}, \\
x_{3}=\hat{H}_{3}, & x_{4}=\hat{H}_{4}, \\
x_{5}=\hat{H}_{5}, & x_{6}=\hat{H}_{6} .
\end{array}
$$

The network is trained on a sample of 4,000 top- pair signal and 3,814 W+jets background events using the six inputs shown above and where both signal and background events have already satisfied the "zero-level" lepton plus missing $E_{T}$ trigger and the calorimeter cell cuts. To get this training sample, it was necessary to generate 50,000 top-pair events and $1,200,000 W+$ jet events.

There is no systematic procedure that provides the best network topology for a given problem. One looks for the simplest network that can discriminate signal from background. Here we use a simple network with only one "hidden layer". We use a 6-12-1 net which has 97 memory parameters (see Ref. [7] for notation). Fig. 11 shows the network response (i.e., $z_{n e t}$ ) for the sample of signal and background events used in the training. The situation is far from the ideal. There are some events around $z_{n e t}=0.5$ for which the net cannot distinguish between signal and background. Nevertheless, the net does allow for some separation of signal and background. The net clearly recognizes some events as signal or background, while for other events there is an overlap and the net cannot distinguish between the two.

The next step is to perform a network cut-off and assign any event with $z_{\text {net }}>z_{\text {cut }}$ to be signal and events with $z_{n e t}<z_{c u t}$ to be background. The enhancement and efficiency of the network cut-off depends on the value chosen for $z_{c u t}$, where the network enhancement and efficiency are defined as follows:

$$
\begin{aligned}
& F_{\text {enh }}^{\text {net }}=\frac{\% \text { of signal with } z_{\text {net }}>z_{\text {cut }}}{\% \text { of background with } z_{\text {net }}>z_{\text {cut }}} \\
& F_{\text {eff }}^{\text {net }}=\% \text { of signal with } z_{\text {net }}>z_{\text {cut }} .
\end{aligned}
$$

The overall network performance can be characterized by the single curve of the network enhancement versus the network efficiency shown in Fig. 12. Each point in Fig. 12 


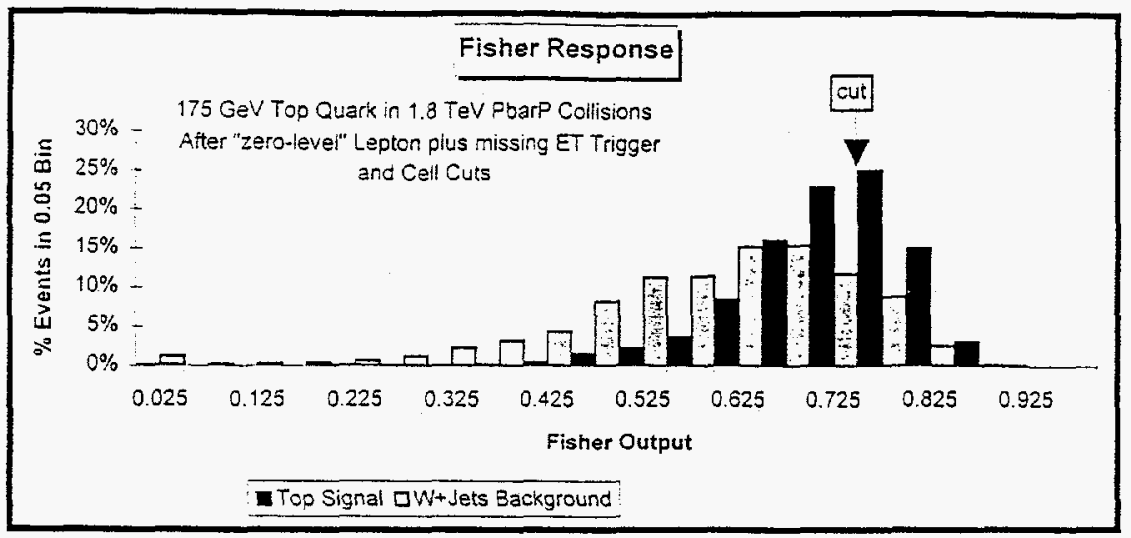

Figure 13 Shows the "shifted" Fisher response, $\hat{F}$, for the sample of signal and background events used in the training of the neural network. The plot corresponds to the percentage of events with $\hat{F}$ within a 0.05 bin for the top-pair signal and the $W$ +jets background. The events have survived the "zero- level" lepton plus missing $E_{T}$ trigger and the calorimeter cell cuts. The position of our "Fisher cut" is marked by the dotted line.

corresponds to a different choice for the network cut-off with the lower efficiencies and higher enhancements corresponding to larger values of $z_{\text {cut }}$. For example, a net cut of $z_{c u t}=0.75$ corresponds to an additional enhancement of about 4 with a relative efficiency of about $47 \%$.

\section{Fisher Discriminates}

A simplier method of separating signal and background is to use Fisher discriminates. This method is analogous to a neural network with no hidden layers. Here as with the network, one inputs a set of $N_{i n}$ variables, $x_{i}$, and there is one output, $F$. However, in this case $F$ is a linear function of the inputs,

$$
F=\sum_{i=1}^{N_{i n}} \alpha_{i} x_{i},
$$

where the Fisher coefficients, $\alpha_{i}$ are chosen to maximize the separation between signal and background in $F$-space,

$$
\frac{\left(\mu_{F}^{s i g}-\mu_{F}^{b a k}\right)^{2}}{\left(\sigma_{F}^{s i g}\right)^{2}+\left(\sigma_{F}^{b a k}\right)^{2}}
$$


where $\mu_{F}$ and $\sigma_{F}$ are the mean and the standard deviation, respectively, of the Fisher output for the signal (sig) and background (bak) sample. The Fisher coefficients are given by

$$
\alpha_{i}=\sum_{j}\left(V^{s i g}+V^{b a k}\right)_{i j}^{-1}\left(\mu_{j}^{s i g}-\mu_{j}^{b a k}\right)
$$

where $\left(V^{s i g}+V^{b a k}\right)^{-1}$ is the inverse matrix and $\mu_{i}$ is the mean of the distribution $x_{i}$,

$$
\mu_{i}=\frac{1}{N} \sum_{n=1}^{N} x_{i}(n)
$$

and $V$ is the covariance matrix,

$$
V_{i j}=\frac{1}{N} \sum_{n=1}^{N}\left(x_{i}(n)-\mu_{i}\right)\left(x_{j}(n)-\mu_{j}\right) .
$$

Here the sum over $n$ corresponds to the sum over the training sample.

In this case training consists of calculating the Fisher coefficients which involves inverting an $N_{i n} \times N_{i n}$ matrix, but this is much easier than training a network. Once this is done the situation is similar to the network (with $F$ replacing $z_{\text {net }}$ ), for each input of $N_{\text {in }}$ variables there is one output $F$. We have determined the Fisher coefficients for the sample of signal and background events used to train our network and the Fisher response for these events is shown in Fig. 13. In plotting the Fisher responce in Fig. 13, we have shifted, $F$, to lie between zero and one as follows:

$$
\hat{F}=\frac{F-F_{\min }}{F_{\max }-F_{\min }} .
$$

In this analysis all the inputs, $x_{i}$, lie between zero and one and all the Fisher coefficients, $\alpha_{i}$, turn our negative which implies that

$$
F_{\max }=0 \quad \text { and } \quad F_{\min }=\sum_{i} \alpha_{i}
$$

The separation between signal and background in Fig. 13 is about the same as the network. As with the network, the overall Fisher performance can be characterized by the single curve of the Fisher enhancement versus the Fisher efficiency which is shown in Fig. 12 


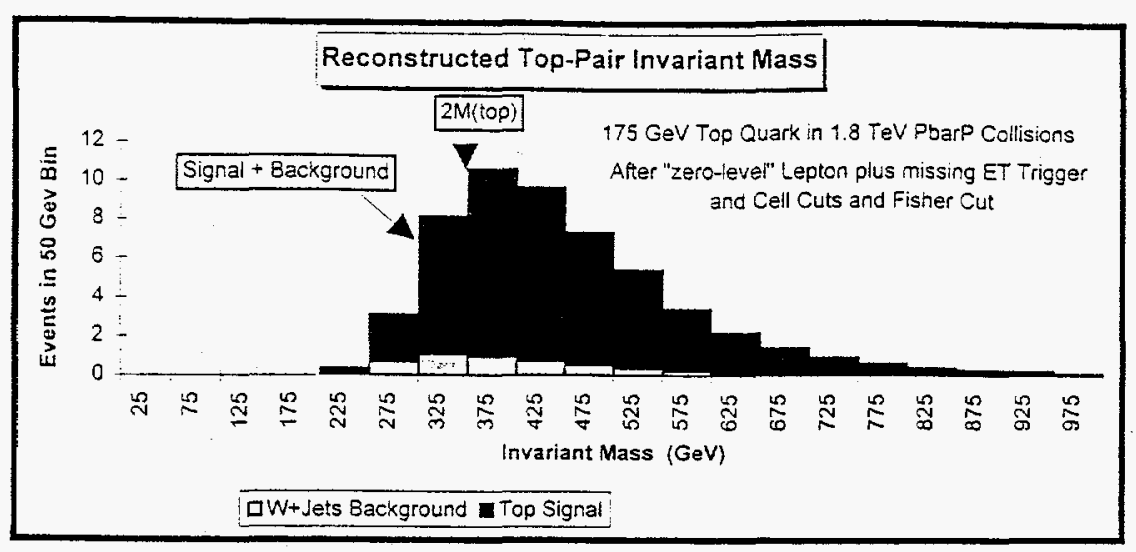

Figure 14 Shows the reconstructed top-pair invariant mass, $M_{t \bar{t}}$, for $175 \mathrm{GeV}$ top quarks produced in $1.8 \mathrm{TeV}$ proton-antiproton collisions together with the $W+$ jets background. The plot shown the sum of the signal plus background and corresponds to the number of events per year (with $\mathcal{L}=100 / \mathrm{pb}$ ) in a $50 \mathrm{GeV}$. The events have survived the "zero-level" lepton plus missing $E_{T}$ trigger, the calorimeter cell cuts, and the Fisher cut-off.

together with the network performance. Each point corresponds to a different choice for the Fisher cut-off.

\section{Using the Fisher Cut-off}

Fig. 12 shown that Fisher discriminates have essencialy the same performance curve as does the neural network and since it is simplier to calculate the Fisher function, we complete our analysis by making a cut on $\hat{F}$ as follows:

- $\hat{F} \geq 0.75$.

As can be seen in Table 1, this Fisher cut provides an additional enhancement of around 4 with a relative efficience of about $44 \%$ resulting in an overall signal to background ratio of about 9 .

Fig. 14 shows the reconstructed parton-parton CM energy for the top-pair signal and the $W$ +jets background after the "zero level" lepton plus missing $E_{T}$ trigger and the calorimeter cell cuts and the Fisher cut. The plot shows the sum of the signal and the background. 


\section{Summary and Conclusions}

We have developed a procedure that enhances the top quark signal $(\ell \nu b \bar{b} q \bar{q}$ decay mode) over the $W+$ jets and the $b \bar{b}+$ jets background in hadron-hadron collisions. Our technique can be summarized by the following series of selections and cuts:

- Lepton and missing transverse energy trigger.

- Calorimeter cell cuts.

- Modified Fox-Wolfram shape parameters (applied to the jets).

- Fisher discriminate or neural network cut-off.

We do not use a conventional "jet trigger". Instead of requiring at least four jets in the event, we find that it is faster and better to simply cut on the number of calorimeter cells with transverse energy greater than some minimum. Our $N_{\text {cell }} \geq 8$ with $E_{T}^{\text {cell }}(\min )=5 \mathrm{GeV}$ cut produces more than a factor of two better enhancement than the traditional, $N_{\text {jet }} \geq 4$, cut. Adding an $E_{T}$ (sum) $>100 \mathrm{GeV}$ cut gives an additional relative enhancement of more than a factor of three.

In addition, we use Neural networks and Fisher discriminates in conjunction with modified Fox-Wolfram "shape" variables to further distinguish the top-pair signal from background. For example, using the first six Fox-Wolfram moments (applied to the jets) together with a Fisher cut-off, $\hat{F} \geq 0.75$, provides an additional enhancement of around 4 with a relative efficiency of about $44 \%$. By combining the calorimeter cell cuts with the event shape information, we are able to obtain an overall signal to background enhancement of around 370 with an efficiency of $30 \%$, and a signal to background ratio of around nine.

\section{References}

1. F. Abe et al. (The CDF Collaboration), Phys. Rev. Lett. 73, 225,1994; Phys. Rev. D50, 2966,1994.

2. S. Abachi et al (The D0 Collaboration), Phys. Rev. Lett. 74, 2632,1995.

3. F. Abe et al. (The CDF Collaboration), Phys. Rev. Lett. 74,2626, 1995.

4. S. Abachi et al (The D0 Collaboration), Phys. Rev. Lett.74, 2422, 1995. 
5. E.W. Varnes (for the D0 Collaboration, presented at 1996 Annual Divisional Meeting (DPF 96) of the Division of Particles and Fields of the American Physical Society, Minneapolis, MN, 10-15 Aug 1996.

6. G. C. Fox and S. Wolfram, Phys. Rev. Lett. 41, 1581 (1978); Nucl. Phys. B149, 413 (1979); Phys. Lett. B82, 134 (1979).

7. R. D. Field, Y. Kanev, M. Tayebnejad, and P. A. Griffin, Phys. Rev. D53, 2296 (1996). 